\title{
Front Matter: Volume 7193
}

, "Front Matter: Volume 7193," Proc. SPIE 7193, Solid State Lasers XVIII: Technology and Devices, 719301 (20 March 2009); doi: 10.1117/12.823717

SPIE Event: SPIE LASE: Lasers and Applications in Science and Engineering, 2009, San Jose, California, United States 


\title{
PROCEEDINGS OF SPIE
}

\section{Solid State Lasers XVIII: Technology and Devices}

\author{
W. Andrew Clarkson \\ Norman Hodgson \\ Ramesh K. Shori \\ Editors
}

25-29 January 2009

San Jose, California, United States

Sponsored by

SPIE

Cosponsored by

Coherent, Inc. (United States)

Published by

SPIE 
The papers included in this volume were part of the technical conference cited on the cover and title page. Papers were selected and subject to review by the editors and conference program committee. Some conference presentations may not be available for publication. The papers published in these proceedings reflect the work and thoughts of the authors and are published herein as submitted. The publisher is not responsible for the validity of the information or for any outcomes resulting from reliance thereon.

Please use the following format to cite material from this book:

Author(s), "Title of Paper," in Solid State Lasers XVIII: Technology and Devices, edited by W. Andrew Clarkson, Norman Hodgson, Ramesh K. Shori, Proceedings of SPIE Vol. 7193 (SPIE, Bellingham, WA, 2009) Article CID Number.

ISSN 0277-786X

ISBN 9780819474391

Published by

SPIE

P.O. Box 10, Bellingham, Washington $98227-0010$ USA

Telephone +1 3606763290 (Pacific Time) · Fax +1 3606471445

SPIE.org

Copyright (c) 2009, Society of Photo-Optical Instrumentation Engineers

Copying of material in this book for internal or personal use, or for the internal or personal use of specific clients, beyond the fair use provisions granted by the U.S. Copyright Law is authorized by SPIE subject to payment of copying fees. The Transactional Reporting Service base fee for this volume is $\$ 18.00$ per article (or portion thereof), which should be paid directly to the Copyright Clearance Center (CCC), 222 Rosewood Drive, Danvers, MA 01923. Payment may also be made electronically through CCC Online at copyright.com. Other copying for republication, resale, advertising or promotion, or any form of systematic or multiple reproduction of any material in this book is prohibited except with permission in writing from the publisher. The CCC fee code is 0277-786X/09/\$18.00.

Printed in the United States of America.

Publication of record for individual papers is online in the SPIE Digital Library.

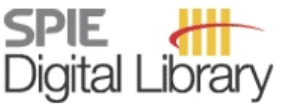

SPIEDigitalLibrary.org

Paper Numbering: Proceedings of SPIE follow an e-First publication model, with papers published first online and then in print and on CD-ROM. Papers are published as they are submitted and meet publication criteria. A unique, consistent, permanent citation identifier (CID) number is assigned to each article at the time of the first publication. Utilization of CIDs allows articles to be fully citable as soon they are published online, and connects the same identifier to all online, print, and electronic versions of the publication. SPIE uses a six-digit CID article numbering system in which:

- The first four digits correspond to the SPIE volume number.

- The last two digits indicate publication order within the volume using a Base 36 numbering system employing both numerals and letters. These two-number sets start with 00, 01, 02, 03, 04 , 05, 06, 07, 08, 09, OA, OB ... 0Z, followed by 10-1Z, 20-2Z, etc.

The CID number appears on each page of the manuscript. The complete citation is used on the first page, and an abbreviated version on subsequent pages. Numbers in the index correspond to the last two digits of the six-digit CID number. 


\section{Contents}

xiii Conference Committee

SESSION 1 SPACE-QUALIFIED LASERS

719304 Some activities of MISSE 6 Mission [7193-03]

N. S. Prasad, NASA Langley Research Ctr. (United States)

719305 Overview of space qualified solid state lasers development at NASA Goddard Space Flight Center [7193-04]

A. W. YU, S. X. Li, G. B. Shaw, A. Seas, M. A. Stephen, E. Troupaki, A. Vasilyev, L. Ramos-lzquierdo, A. Lukemier, W. Mamakos, A. Melak, J. Guzek, A. Rosanova, NASA Goddard Space Flight Ctr. (United States)

719306 Longevity validation of the LOLA laser design by extended vacuum testing of the LOLA engineering model laser [7193-05]

G. B. Shaw, M. A. Stephen, E. Troupaki, A. A. Vasilyev, A. W. Yu, NASA Goddard Space Flight Ctr. (United States)

719307 Qualification of laser diode arrays for space applications [7193-06]

E. Troupaki, A. A. Vasilyev, Science Systems \& Applications, Inc. (United States);

M. A. Stephen, A. A. Seas, N. B. Kashem, NASA Goddard Space Flight Ctr. (United States)

\section{SESSION 2 HIGH POWER PULSED LASERS}

719309 Q-switched Nd:YAG lasers for high average-power and high peak-power operation [7193-09]

M. Poulter, N. Hay, B. Fulford, P. Campton, M. Mason, Powerlase Ltd. (United Kingdom);

D. Burns, Univ. of Strathclyde (United Kingdom)

$71930 \mathrm{~A}$ Power-scaling and prototyping of a gain switched Ti:Sapphire laser with a tuning range of $400 \mathrm{~nm}$ [7193-10]

B. Jungbluth, D. Oberbeckmann, J. Wueppen, Fraunhofer-Institut für Lasertechnik (Germany)

7193 OC Slab lasers and second harmonic generation in near field [7193-12]

D. Li, P. Zhu, Z. Ma, R. Haas, A. Schell, P. Shi, K. Du, EdgeWave GmbH (Germany)

SESSION 3 HIGH POWER BEAM DELIVERY AND COMBINING

7193 OD Photothermal common-path interferometry (PCI): new developments (Invited Paper) [7193-13]

A. Alexandrovski, Stanford Thermal Solutions (United States); M. Fejer, A. Markosian, R. Route, Stanford Univ. (United States) 
7193 OE Characterizing high power laser beams to detect the thermal load of optics and to identify limitations within the design of the optical system [7193-14]

H. Schwede, O. Märten, R. Kramer, S. Wolf, V. Brandl, K. Hänsel, PRIMES GmbH (Germany)

7193 OF Fundamental-mode fiber-to-fiber coupling at high-power [7193-15]

M. Blomqvist, M. Pålsson, O. Blomster, Optoskand AB (Sweden); G. Manneberg, AlbaNova Univ. Ctr., Royal Institute of Technology (Sweden)

\section{SESSION 4 YTTERBIUM LASERS}

7193 Ol High power multi-crystal cw and femtosecond mode-locked oscillators based on Yb:KYW [7193-21]

K. Wentsch, A.-L. Calendron, M. J. Lederer, High Q Laser Innovation GmbH (Austria)

7193 OJ Diode-pumped Yb:KYW laser emitting at $981 \mathbf{n m}$ by intracavity pumping [7193-22]

M. Castaing, Lab. Charles Fabry de l'Institut d'Optique, CNRS, Univ. Paris Sud (France) and Oxxius S.A. (France); F. Balembois, P. Georges, Lab. Charles Fabry de I'Institut d'Optique, CNRS, Univ. Paris Sude (France); T. Georges, Oxxius S.A. (France)

7193 OK Power scaling of ytterbium INNOSLAB amplifiers beyond 100W average power [7193-23] P. Rußbüldt, H.-D. Hoffmann, T. G. Mans, Fraunhofer-Institut für Lasertechnik (Germany)

7193 OL Diode-pumped laser with Yb:YAG single-crystal fiber grown by the micro-pulling down technique [7193-24]

D. Sangla, Lab. Charles Fabry de I'Institut d'Optique, CNRS, Univ Paris Sud (France) and Univ. de Claude Bernard Lyon I, CNRS, Lab. de Physico-Chimie des Matériaux Luminescents (France); N. Aubry, Univ. de Claude Bernard Lyon I, CNRS, Lab. de Physico-Chimie des Matériaux Luminescents (France) and Fibercryst SAS (France); J. Didierjean, Lab. Charles Fabry de l'Institut d'Optique, CNRS, Univ Paris Sud (France); D. Perrodin, FiberCryst SAS (France); F. Balembois, Lab. Charles Fabry de I'Institut d'Optique, CNRS, Univ Paris Sud (France); K. Lebbou, A. Brenier, Univ. de Claude Bernard Lyon I, CNRS, Lab. de Physico-Chimie des Matériaux Luminescents (France); P. Georges, Lab. Charles Fabry de I'Institut d'Optique, CNRS, Univ Paris Sud (France); J. Fourmigué, FiberCryst SAS (France); O. Tillement, Univ. de Claude Bernard Lyon I, CNRS, Lab. de Physico-Chimie des Matériaux Luminescents (France)

\section{SESSION 5 VANADATE LASERS}

$71930 \mathrm{M} \quad$ Comparative studies of $\mathrm{Nd}: \mathrm{Y}_{\mathbf{x}} \mathbf{G d}_{1-\mathrm{x}} \mathbf{V O}_{4}$ laser with direct and indirect pumping [7193-25] N. C. Fernelius, Air Force Research Lab. (United States); X. Wang, Y. Tang, S. Tang, Crystal Research, Inc. (United States)

$7193 \mathrm{ON}$ Three-level operation in a diode-pumped Nd:GdVO 4 laser and CW 440-nm generation [7193-26]

M. Castaing, Lab. Charles Fabry de l'Institut d'Optique, CNRS (France) and Oxxius S.A. (France); F. Balembois, P. Georges, Lab. Charles Fabry de I'Institut d'Optique, CNRS, Univ. Paris Sud (France) 
719300 Optimization of a high-power fiber-coupled diode-end-pumped $\mathrm{Nd}^{\mathrm{YVO}_{4}}$ solid state laser [7193-27]

A. F. El-Sherif, Egyptian Armed Forces (Egypt)

$7193 \mathrm{OP}$ A mode-hop-free tunable single-longitudinal-mode $\mathrm{Nd}: \mathrm{YVO}_{4}$ laser with $25 \mathrm{~W}$ of power at 1064nm [7193-28]

K. M. Murdoch, D. A. Clubley, M. J. Snadden, Coherent, Inc. (United States)

$7193 \mathrm{OQ}$ Lasing of $\mathrm{Yb}$ in a mixed $\mathrm{YGdVO}_{4}$ crystal with bistability and polarization switching [7193-29]

J. Liu, Qingdao Univ. (China); H. Zhang, Shandong Univ. (China); X. Mateos, Univ. Rovira

i Virgili (Spain); W. Han, Qingdao Univ. (China); V. Petrov, Max-Born-Institute for Nonlinear

Optics and Ultrafast Spectroscopy (Germany)

\section{SESSION 6 STABILIZED LASERS}

7193 OR Spectral narrowing and tunability of a high-power diffraction-limited ns-pulsed OPO/OPA system using transversely chirped and temperature-tuned volume Bragg gratings [7193-30] A. J. Merriam, Lumeras LLC (United States); V. Smirnov, L. Glebov, OptiGrate (United States)

7193 OS Injection seeded single frequency Nd:YAG MOPA Q-switched laser [7193-31]

F. F. Wu, A. Khizhnyak, V. Markov, MetroLaser, Inc. (United States)

7193 OT Single-frequency glass waveguide lasers [7193-32]

S. Taccheo, Swansea Univ. (United Kingdom); G. Della Valle, Politecnico di Milano (Italy);

D. Barbier, Teemphotonics (France)

7193 OU Narrow linewidth tunable CW thulium fiber lasers with VBG and GMRF stabilization [7193-33] R. A. Sims, T. McComb, V. Sudesh, M. Reichert, M. Richardson, College of Optics \& Photonics, Univ. of Central Florida (United States); M. Poutous, Z. Roth, E. G. Johnson, Univ. of North Carolina at Charlotte (United States)

7193 OV A novel tunable diode laser using volume holographic gratings [7193-34]

C. Moser, L. Ho, F. Havermeyer, Ondax, Inc. (United States)

\section{SESSION $7 \quad$ VISIBLE AND UV LASERS I: JOINT SESSION WITH CONFERENCE 7195}

7193 OW Highly stable UV-mode-locked lasers with an output power of $35 \mathbf{W}$ at $355 \mathbf{~ n m ~ [ 7 1 9 3 - 3 5 ] ~}$ M. Mond, H. Schoene, Coherent Lübeck GmbH (Germany); A. Diening, Coherent Inc. (United States); G. Hollemann, W. Seelert, Coherent Lübeck GmbH (Germany)

7193 0X Nanosecond 389-nm coherent light source with injection seeding for nuclear spin polarization of ${ }^{3} \mathrm{He}$ atoms [7193-36]

Y. Aoki, Y. Shiomi, T. Yamamoto, H. Kumagai, A. Kobayashi, Osaka City Univ. (Japan)

7193 OY High power UV generation at $355 \mathrm{~nm}$ by means of extracavity frequency conversion of a high repetition rate Innoslab MOPA system [7193-37]

B. Gronloh, M. Höfer, R. Wester, H.-D. Hoffmann, Fraunhofer-Institut für Lasertechnik (Germany) 
SESSION $8 \quad$ VISIBLE AND UV LASERS II: JOINT SESSION WITH CONFERENCE 7197

$71930 Z$ Q-switched diode-pumped Nd:YAG rod laser with output power of $420 \mathrm{~W}$ at $532 \mathrm{~nm}$ and $160 \mathrm{~W}$ at $355 \mathrm{~nm}$ [7193-38]

D. R. Dudley, O. Mehl, G. Y. Wang, E. S. Allee, H. Y. Pang, N. Hodgson, Coherent, Inc. (United States)

\section{SESSION 9 OPS AND VECSELS I}

719311 Recent developments in high-power short-wave mid-infrared semiconductor disk lasers (Invited Paper) [7193-40]

D. Burns, J.-M. Hopkins, A. J. Kemp, Univ. of Strathclyde (United Kingdom); B. Rösener, N. Schulz, C. Manz, K. Köhler, M. Rattunde, J. Wagner, Fraunhofer-Institut für Angewandte Festkörperphysik (Germany)

719313 OPS laser EPI design for different wavelengths (Invited Paper) [7193-42]

J. V. Moloney, J. Hader, Nonlinear Control Strategies (United States) and College of Optical Sciences, Univ. of Arizona (United States); H. Li, Y. Kaneda, T. S. Wang, M. Yarborough, College of Optical Sciences, Univ. of Arizona (United States); S. W. Koch, Philipps-Univ. Marburg (Germany); W. Stolz, B. Kunert, NAsP GmbH (Germany) and Philipps-Univ. Marburg (Germany); C. Bueckers, S. Chaterjee, Philipps-Univ. Marburg (Germany); G. Hardesty, College of Optical Sciences, Univ. of Arizona (United States)

719314 Aberration-insensitive resonators for OPS lasers [7193-43]

A. Caprara, Coherent, Inc. (United States)

SESSION 10 OPS AND VECSELS II

$719316 \quad 1220$ nm mode-locked GalnNAs disk laser [7193-45]

M. Guina, J. Rautiainen, V.-M. Korpijärvi, J. Puustinen, O. Okhotnikov, Tampere Univ. of Technology (Finland)

719317 GalnNAs semiconductor disk lasers as pump sources for $\operatorname{Tm}^{+}\left(, \mathrm{Ho}^{3+}\right)$-doped glass, crystal and fibre lasers [7193-47]

S. L. Vetter, L. J. McKnight, S. Calvez, M. D. Dawson, Univ. of Strathclyde (United Kingdom); F. Fusari, A. A. Lagatsky, W. Sibbett, C. T. A. Brown, Univ. of St Andrews (United Kingdom); V.-M. Korpijärvi, M. Guina, Tampere Univ. of Technology (Finland); B. Richards, G. Jose, A. Jha, Univ. of Leeds (United Kingdom)

\section{SESSION 11 SEMICONDUCTOR LASERS WITH HARMONIC GENERATION}

719318 Deep-ultraviolet frequency converted optically pumped semiconductor laser (Invited Paper) [7193-48]

Y. Kaneda, T.-L. Wang, J. M. Yarborough, M. Fallahi, J. V. Moloney, College of Optical Sciences, Univ. of Arizona (United States); M. Yoshimura, Y. Mori, T. Sasaki, Osaka Univ. (Japan) 
719319 Intracavity-tripled optically pumped semiconductor laser at $\mathbf{3 5 5} \mathbf{~ n m ~ [ 7 1 9 3 - 4 9 ] ~}$ Q.-Z. Shu, A. L. Caprara, J. D. Berger, D. W. Anthon, H. Jerman, L. Spinelli, Coherent, Inc. (United States)

$71931 \mathrm{~A} \quad$ Ultra-narrow bandwidth OPS laser in the green-yellow wavelength range for Raman spectroscopy [7193-50]

C. Kannengiesser, W. Seelert, V. Ostroumov, R. von Elm, S. Hilbich, M. Bracker, Coherent Lübeck GmbH (Germany); J. Lindfors, Coherent Finland Oy (Finland)

7193 1C Compact Watt-class visible light sources using direct frequency-doubled edge-emitting diode lasers [7193-52]

K. Paschke, G. Blume, C. Fiebig, A. Sahm, D. Feise, M. Uebernickel, G. Ebert, G. Tränkle, Ferdinand-Braun-Institut für Höchstfrequenztechnik (Germany)

7193 ID High-power pulsed intra-cavity frequency doubled vertical extended cavity blue laser arrays [7193-53]

R. van Leeuwen, J.-F. Seurin, G. Xu, C. Ghosh, Princeton Optronics, Inc. (United States)

$71931 \mathrm{E} \quad$ Compact blue light sources based on grating coupled surface emitting lasers [7193-54] O. V. Smolski, Y. O. Yilmaz, V. O. Smolski, E. G. Johnson, The Univ. of North Carolina at Charlotte (United States)

7193 IF GaSb-based compounds tailored for MID-IR disk lasers (Invited Paper) [7193-55] M. Guina, A. Härkönen, S. Suomalinen, J. Paajaste, R. Koskinen, M. Pessa, O. Okhotnikov, Tampere Univ. of Technology (Finland)

$71931 \mathrm{G}$ Lead-chalcogenide VECSELs on Si and $\mathrm{BaF}_{2}$ for $5 \boldsymbol{\mu m}$ emission (Invited Paper) [7193-56] M. Rahim, A. Khiar, F. Felder, M. Fill, D. Boye, H. Zogg, ETH Zürich (Switzerland)

$71931 \mathrm{H} \quad$ Tm:fiber laser in-band pumping a cryogenically cooled Ho:YAG laser [7193-57] J. I. Mackenzie, W. O. S. Bailey, J. W. Kim, L. Pearson, D. Y. Shen, Y. Yang, W. A. Clarkson, Univ. of Southampton (United Kingdom)

$719311 \quad$ Widely tunable (>100 nm) continuous-wave narrow-linewidth high-power thulium fiber laser [7193-58]

T. S. McComb, V. Sudesh, L. Shah, R. A. Sims, M. C. Richardson, College of Optics \& Photonics, Univ. of Central Florida (United States)

$71931 \mathrm{~J} \quad$ 10-watt room-temperature Er-fiber-laser-pumped pure CW polycrystalline $\mathrm{Cr}^{2+}: \mathrm{ZnS}$ laser [7193-59]

I. S. Moskalev, V. V. Fedorov, S. B. Mirov, Univ. of Alabama at Birmingham (United States)

$71931 \mathrm{~K} \quad$ Bulk Fe:ZnSe laser gain-switched by the Q-switched Er:YAG laser [7193-60]

M. E. Doroshenko, T. T. Basiev, General Physics Institute (Russian Federation); P. Koranda, H. Jelínková, M. Nĕmec, M. Čech, J. Šulc, Czech Technical Univ. in Prague (Czech Republic); V. K. Komar, A. S. Gerasimenko, Institute for Single Crystals (Ukraine); V. V. Badikov, D. V. Badikov, Kuban State Univ. (Russian Federation) 
$71931 \mathrm{~L} \quad$ High-energy ultrafast thin-disk oscillators (Invited Paper) (Best Student Paper Award) [7193-62]

J. Neuhaus, D. Baver, TRUMPF Laser GmbH \& Co. KG (Germany) and Univ. of Konstanz (Germany); C. Scharfenberg, D. H. Sutter, J. Kleinbaver, S. Weiler, A. Killi, TRUMPF Laser GmbH \& Co. KG (Germany); T. Dekorsy, Univ. of Konstanz (Germany)

7193 1M Compact cavity dumped broadband laser oscillator [7193-63]

E. Coadou, Coherent, Inc. (United States); D. Neumeyer, APE GmbH (Germany); B. Resan, A. Schill, Coherent, Inc. (United States); I. Rimke, APE GmbH (Germany)

$71931 \mathrm{P}$ Short-pulse-OPO for near-infrared bandwidths up to $150 \mathrm{~nm}$ [7193-66] E. Buettner, I. Rimke, APE GmbH (Germany); R. Viselga, W. Tulloch, Coherent, Inc. (United States); G. Stibenz, APE GmbH (Germany);

$71931 Q \quad$ Light-matter interaction processes behind intra-cavity mode-locking devices [7193-67] C. Roychoudhuri, Univ. of Connecticut (United States) and Femto Macro Continuum (United States); N. S. Prasad, NASA Langley Research Ctr. (United States)

\section{SESSION 14 DISK LASERS}

7193 IR Disk laser: a new generation of industrial lasers [7193-68]

R. Brockmann, TRUMPF Laser GmbH \& Co. KG (Germany); D. Havrilla, TRUMPF, Inc. (United States)

7193 is $\quad 50-k H z, 400-\mu \mathrm{J}$, sub-100-fs pulses from a thin disk laser amplifier [7193-69] M. Larionov, Technologiegesellschaft für Strahlwerkzeuge mbH (Germany); A. Giesen, Institut fur Strahlwerkzeuge, Univ. Stuttgart (Germany)

7193 IT The broad applicability of the disk laser principle: from CW to ps [7193-70] A. Killi, C. Stolzenburg, I. Zawischa, D. Sutter, J. Kleinbauer, S. Schad, R. Brockmann, S. Weiler, TRUMPF Laser GmbH \& Co. KG (Germany); J. Neuhaus, Univ. of Konstanz (Germany); S. Kalfhues, E. Mehner, TRUMPF Laser GmbH \& Co. KG (Germany); D. Baver, TRUMPF Laser GmbH \& Co. KG (Germany) and Univ. Of Konstanz (Germany); H. Schlveter, Technolas Perfect Vision GmbH (Germany); C. Schmitz, TRUMPF Laser GmbH \& Co. KG (Germany)

$71931 \mathrm{U}$ Quenching processes in Yb lasers: correlation to the valence stability of the $\mathrm{Yb}$ ion [7193-71] M. Engholm, Fiber Optic Valley AB (Sweden); L. Norin, Acreo FiberLab (Sweden); C. Hirt, S. T. Fredrich-Thornton, K. Petermann, G. Huber, Univ. Hamburg (Germany)

$71931 \mathrm{~V}$ Thin disk laser: power scaling to the $\mathrm{kW}$ regime in fundamental mode operation [7193-72] J. Mende, E. Schmid, J. Speiser, G. Spindler, A. Giesen, Institute of Technical Physics, German Aerospace Ctr. (Germany) 
$71931 \mathrm{X}$ Development of ceramic solid-state laser host materials [7193-74]

N. S. Prasad, NASA Langley Research Ctr. (United States); S. Trivedi, S. Kutcher, C.-C. Wang, J.-S. Kim, Brimrose Corp. of America (United States); U. Hommerich, Hampton Univ. (United States); V. Shukla, R. Sadangi, Rutgers Univ. (United States)

71931 1Y Single-frequency Nd:YGG laser at $935 \mathrm{~nm}$ for future water-vapour DIAL systems (Best Student Paper Award) [7193-75]

J. Löhring, A. Meissner, V. Morasch, P. Becker, Fraunhofer-Institut für Lasertechnik (Germany); W. Heddrich, Hochschule Darmstadt (Germany); D. Hoffmann, Fraunhofer-Institut für Lasertechnik (Germany)

719320 Quasi-continuously pumped operation of $2.4 \%$ doped crystalline Nd:YAG in a bounce geometry [7193-78]

V. Kubeček, M. Jelínek, M. Čech, P. Hiršl, Czech Technical Univ. in Prague (Czech Republic)

\section{SESSION 16 APPLICATIONS I}

719321 Selective removal of dielectric layers using picoseconds UV pulses (Invited Paper) [7193-80] V. V. Rana, Z. Zhang, Applied Materials, Inc. (United States)

719322 Comparison of $n s$ and ps pulses for Si and glass micromachining applications [7193-81] S. Lee, A. Ashmead, L. Migliore, Coherent, Inc. (United States)

719324 Generation of programmable temporal pulse shape and applications in micromachining [7193-93]

X. Peng, B. Jordens, A. Hooper, B. W. Baird, W. Ren, L. XU, L. Sun, Electro Scientific Industries, Inc. (United States)

\section{SESSION 17 APPLICATIONS II}

719325 Ultrafast optical parametric oscillators for spectroscopy (Invited Paper) [7193-84] D. T. Reid, Ł. W. Kornaszewski, T. P. Müller, N. Gayraud, W. N. MacPherson, D. P. Hand, Heriot-Watt Univ. (United Kingdom); J. M. Stone, J. C. Knight, Univ. of Bath (United Kingdom)

719326 Multimodal ultrafast spectroscopy system based on 35 fs Ti:Sapphire CPA laser [7193-85] R. Zadoyan, Newport Corp. (United States)

719327 Wide horizons for optical trapping: simultaneous optical manipulation and confocal imaging of live T cells at low magnification [7193-86]

J. Harris, G. McConnell, Univ. of Strathclyde (United Kingdom)

719328 Solid state lasers for wide-field CARS microscopy [7193-87]

D. Simanovskii, I. Toytman, Stanford Univ. (United States); D. Palanker, Stanford Univ. (United States) and Stanford Univ. School of Medicine (United States) 
7193 2A Industrial applications of high-average power high-peak power nanosecond pulse duration Nd:YAG lasers (Invited Paper) [7193-89]

P. M. Harrison, S. Ellwi, Powerlase Ltd. (United Kingdom)

7193 2B Unique performances and favourable applications of INNOSLAB lasers [7193-90]

K. Du, EdgeWave GmbH (Germany)

\section{POSTER SESSION}

71932 2E Design of PETAL project main amplifier [7193-95]

T. Berthier, N. Blanchot, L. Espert, J. P. Lasserre, E. Perrot Minnot, A. Roques, Commissariat à l'Energie Atomique (France)

$71932 \mathrm{~F} \quad$ Study of optical centers with near-infrared emission in germanate glasses doped with $6 \rho$ (Bi, $\mathrm{Pb})$ and $5 \rho(\mathrm{Sn}, \mathrm{Sb})$ ions [7193-97]

M. Sharonov, A. Bykov, R. R. Alfano, The City College of New York (United States)

$71932 \mathrm{H} \quad$ High-efficient room-temperature CW operating Tm:YAP laser with microchip resonator [7193-99]

J. Šulc, H. Jelínková, Czech Technical Univ. in Prague (Czech Republic); K. Nejezchleb, V. Škoda, Crytur Ltd. (Czech Republic)

$71932 \mathrm{~J} \quad$ Continuous-wave and Q-switched laser operation of the disordered $\mathrm{Yb}: \mathrm{Ca}_{3}(\mathrm{NbGa})_{2-x} \mathrm{Ga}_{3} \mathrm{O}_{12}$ crystal [7193-101]

J. Liu, W. Han, Qingdao Univ. (China); H. Zhang, J. Wang, Shandong Univ. (China); V. Petrov, Max-Born-Institute for Nonlinear Optics and Ultrafast Spectroscopy (Germany)

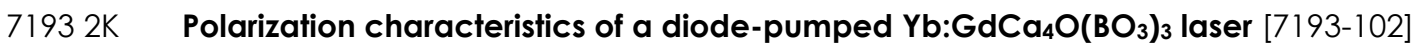
J. Liu, H. Yang, Qingdao Univ. (China); H. Zhang, J. Wang, Shandong Univ. (China); V. Petrov, Max-Born-Institute for Nonlinear Optics and Ultrafast Spectroscopy (Germany)

$71932 \mathrm{M}$ Compact, rigid, and high-power ultrafast laser system applying a glass-block cavity [7193-104]

S. Aoshima, S. Oishi, T. Moriguchi, Y. Kawada, M. Fujimoto, K. Shibayama, M. Takasaka, K. Fukumitsu, S. Sakamoto, K. Yamamoto, Hamamatsu Photonics K.K. (Japan)

7193 2N Flash-lamp pumped Pr:YAP laser operated at wavelengths of $747 \mathrm{~nm}$ and $662 \mathrm{~nm}$ (Best Student Poster Award) [7193-106]

M. Fibrich, H. Jelínková, J. Šulc, Czech Technical Univ. in Prague (Czech Republic);

K. Nejezchleb, V. Škoda, Crytur Ltd. (Czech Republic)

719320 Analytical model of passively Q-switched Nd:YAG/V:YAG microchip laser [7193-107] J. Šulc, H. Jelínková, Czech Technical Univ. in Prague (Czech Republic)

$71932 \mathrm{P} \quad$ Resonantly pumped Er:YAG and Er:YAP lasers [7193-108]

M. Nĕmec, H. Jelínková, J. Šulc, Czech Technical Univ. in Prague (Czech Republic);

K. Nejezchleb, V. Škoda, Crytur, Ltd. (Czech Republic) 
$71932 \mathrm{Q} \quad$ Q-switched hybrid MOPA laser system based on Yb fibre with side pumping by single source [7193-109]

S. Kobtsev, S. Kukarin, Y. Fedotov, Novosibirsk State Univ. (Russian Federation)

$71932 \mathrm{R} \quad$ Chromium doped ZnSe and ZnS gain media for optically and electrically pumped mid-IR lasers [7193-110]

C. Kim, J. M. Peppers, D. V. Martyshkin, V. V. Fedorov, S. B. Mirov, Univ. of Alabama at Birmingham (United States)

$719325 \quad$ Wide-autoscanned narrow-line tunable system based on CW Ti:Sapphire/dye laser for high-precision experiments in nanophysics [7193-111]

S. Kobtsev, Novosibirsk State Univ. (Russian Federation) and Tekhnoscan JSC (Russian Federation); V. Baraulya, Tekhnoscan JSC (Russian Federation); V. Lunin, Novosibirsk State Univ. (Russian Federation) and Tekhnoscan JSC (Russian Federation)

Author Index 
Downloaded From: https://www.spiedigitallibrary.org/conference-proceedings-of-spie on 26 Apr 2023

Terms of Use: https://www.spiedigitallibrary.org/terms-of-use 


\title{
Conference Committee
}

\author{
Symposium Chairs
}

Donald J. Harter, IMRA America, Inc. (United States)

Peter R. Herman, University of Toronto (Canada)

Symposium Cochairs

Henry Helvajian, The Aerospace Corporation (United States)

Friedrich G. Bachmann, Rofin-Sinar Laser GmbH (Germany)

Program Track Chair

Gregory J. Quarles, VLOC (United States)

Conference Chairs

W. Andrew Clarkson, University of Southampton (United Kingdom)

Norman Hodgson, Coherent, Inc. (United States)

Ramesh K. Shori, Naval Air Warfare Center (United States)

Program Committee

Martin D. Dawson, University of Strathclyde (United Kingdom)

Adolf Giesen, Deutsches Zentrum für Luft- und Raumfahrt e.V. (Germany)

Hanna J. Hoffman, Electro Optics Organization, Inc. (United States)

Hans-Dieter Hoffmann, Fraunhofer-Institut für Lasertechnik (Germany)

Helena Jelínková, Czech Technical University in Prague (Czech Republic)

Jacob I. Mackenzie, University of Southampton (United Kingdom) Christine Klemenz, University of Central Florida (United States)

Jerome V. Moloney, College of Optical Sciences, The University of Arizona (United States)

Michio Oka, Sony Corporation (Japan)

Alan B. Petersen, Spectra-Physics (United States)

Stephen G. Post, Missile Defense Agency (United States)

Narasimha S. Prasad, NASA Langley Research Center (United States)

Martin C. Richardson, College of Optics \& Photonics, University of Central Florida (United States)

Wolf R. Seelert, Coherent Lübeck GmbH (Germany)

Irina T. Sorokina, Norwegian University of Science and Technology (Norway)

David H. Titterton, Defence Science and Technology Laboratory

(United Kingdom) 
Session Chairs

1 Space-qualified Lasers

Ramesh K. Shori, Naval Air Warfare Center (United States)

2 High Power Pulsed Lasers

Narasimha S. Prasad, NASA Langley Research Center (United States)

$3 \quad$ High Power Beam Delivery and Combining

Helena Jelínková, Czech Technical University in Prague (Czech Republic)

$4 \quad$ Ytterbium Lasers

Martin C. Richardson, College of Optics \& Photonics, University of Central Florida (United States)

5 Vanadate Lasers

Alan B. Petersen, Newport Spectra-Physics (United States)

6 Stabilized Lasers

Wolf R. Seelert, Coherent Lübeck GmbH (Germany)

7 Joint Session with Conference 7195: Visible and UV Lasers I

Norman Hodgson, Coherent, Inc. (United States)

Dahv A. V. Kliner, JDS Uniphase Corp. (United States)

8 Joint Session with Conference 7197: Visible and UV Lasers II

W. Andrew Clarkson, University of Southampton (United Kingdom)

Pinhas Blau, Soreq Nuclear Research Center (Israel)

9 OPS and VECSELS I

Adolf Giesen, Deutsches Zentrum für Luft- und Raumfahrt e.V.

(Germany)

10 OPS and VECSELS II

Jerome V. Moloney, College of Optical Sciences, The University of Arizona (United States)

11 Semiconductor Lasers with Harmonic Generation

David Burns, University of Strathclyde (United Kingdom)

12 Mid-Infrared Lasers

Ramesh K. Shori, Naval Air Warfare Center (United States)

13 Ultrafast Lasers

W. Andrew Clarkson, University of Southampton (United Kingdom) 
14 Disk Lasers

Hans-Dieter Hoffmann, Fraunhofer-Institut für Lasertechnik (Germany)

15 Laser Materials

Jacob I. Mackenzie, University of Southampton (United Kingdom)

16 Applications I

Norman Hodgson, Coherent, Inc. (United States)

17 Applications II

David H. Titterton, Defence Science and Technology Laboratory (United Kingdom)

18 Applications III

Helena Jelínková, Czech Technical University in Prague (Czech Republic)

Best Student Presentation Awards Ceremony

Norman Hodgson, Coherent, Inc. (United States) 
Downloaded From: https://www.spiedigitallibrary.org/conference-proceedings-of-spie on 26 Apr 2023

Terms of Use: https://www.spiedigitallibrary.org/terms-of-use 